\title{
An introductory overview of orbital tumors
}

\author{
Tim E. Darsaut, B.Sc.H., Giuseppe Lanzino, M.D., M. Beatriz Lopes, M.D., AND \\ STEVEn NeWMan, M.D. \\ Departments of Neurosurgery and Ophthalmology, and Division of Neuropathology, University of \\ Virginia Health Sciences Center, Charlottesville, Virginia
}

\begin{abstract}
The term "orbital tumors" comprises a wide variety of lesions that often share the same cardinal clinical finding (exophthalmos) and clinical history. Age at presentation, associated ophthalmological findings, and radiological features, however, provide invaluable information as to the possible histological type of tumor. The present article serves as an introductory overview regarding the pathological characteristics, clinical features, radiological characteristics, and principles of treatment of orbital tumors.
\end{abstract}

\section{KEY WORDS • orbital tumor • exophthalmos • hemangioma • meningioma • schwannoma • optic glioma • metastasis}

Orbital tumors constitute a heterogeneous array of lesions (Table 1) and, as such, pose numerous challenges in terms of diagnosis, imaging and management. ${ }^{2,5}$ Given the variety of structures within the relatively confined orbit, a systematic approach is necessary to understand the classification and clinical features of orbital tumors. Schematically, orbital tumors can be classified based on origin: 1) primary lesions, which originate from the orbit itself; 2) secondary lesions, which extend to the orbit from neighboring structures and include such lesions as intracranial tumors and tumors of the paranasal sinuses that, by contiguity, extend to involve the orbit; and 3) metastatic tumors. Orbital tumors are also divided anatomically into intraconal and extraconal depending on their relationship with the muscle cone. The muscle cone is formed by the extraocular rectus muscles and their intermuscular septae, which separate the intraconal from the extraconal space. The muscle cone has a conical shape with the globe serving as the base and the optic canal as its apex.

In the present article, we provide a general overview of the pathological characteristics, clinical features, and imaging characteristics of primary orbital tumors. Metastatic lesions are also briefly reviewed.

\section{OVERVIEW OF ORBITAL TUMORS}

\section{Tumor Arising From Bone and Cartilaginous Structures}

The frontal, zygomatic, maxillary, sphenoid, lacrimal,

Abbreviations used in this paper: AVM = arteriovenous malformation; $\mathrm{CT}=$ computerized tomography; $\mathrm{MR}=$ magnetic resonance; $\mathrm{NF}=$ neurofibromatosis; NF1 = NF Type 1 . palatine, and ethmoid bones bones form the orbit. The bony orbit protects the globe and allows for passage of the optic nerve, the ophthalmic division of the trigeminal nerve, the ocular motor nerves (third, fourth, and sixth), and the vessels required for proper function of the eye. These bones may be involved in fibroosseous tumor growth, such as osteomas, ossifying fibromas, fibrous dysplasia, aneurysmal bone cysts, and osteogenic sarcoma.

Osteomas arise most commonly from anterior ethmoid cells, although they are known to originate from posterior ethmoidal cells and sphenoid cells. This tumor exhibits a slowly invading growth pattern, and it can be associated with the presence of a mucocele if normal sinus drainage is occluded. Ossifying fibromas are benign, progressive, recurrent tumors that cause painless facial swelling and displacement of the eye; because of their possible extension into the anterior and middle cranial fossae, they can be problematic. These lesions are readily identified on CT scans by their diffusely dense signal.

Fibrous dysplasia commonly involves the sphenoid or frontal bone. It produces a general expansion of the involved bones and does not form a definable soft-tissue mass. Pathologically, they contain fibrous stroma with patches of osteoid but no osteoblasts (Fig. 1). This characteristic leads to a low-intensity signal on $\mathrm{T}_{1}$ - and $\mathrm{T}_{2}-$ weighted MR images. On administration of gadolinium, however, they show enhancement. ${ }^{7}$ The lack of osteoblasts prevents proper bone formation, and the lesion becomes clinically significant by either encroaching on the optic canal or by causing cosmetic disturbances.

Aneurysmal bone cysts commonly affect the frontal bone in young children. These cysts form a large cavernous space filled with blood. It is thought that the inflam- 
TABLE 1

Summary of 1376 orbital lesions according to major category*

\begin{tabular}{|c|c|c|c|}
\hline \multirow[b]{2}{*}{ Category } & \multicolumn{3}{|c|}{ No. of Lesions (\%) } \\
\hline & Primary & Secondary & Total \\
\hline osseous and cartilagenous & & & $42(3)$ \\
\hline osteoma & 3 & 7 & 10 \\
\hline osteogenic sarcoma & 2 & 11 & 13 \\
\hline chondroma & 1 & & 1 \\
\hline chondrosarcoma & 1 & 5 & 6 \\
\hline fibrous dysplasia & 5 & 1 & 6 \\
\hline aneurysmal bone cyst & 2 & & 2 \\
\hline vascular & & & $131(9.5)$ \\
\hline capillary hemangioma & 30 & & 30 \\
\hline cavernous hemangioma & 60 & & 60 \\
\hline hamartoma & 1 & & 1 \\
\hline hemangiopericytoma & 16 & & 16 \\
\hline lymphangioma & 18 & & 18 \\
\hline nerve sheath & & & $54(3.9)$ \\
\hline neurolemmoma & 15 & & 15 \\
\hline neurofibroma (plexiform) & 9 & & 9 \\
\hline neurofibroma (solitary) & 8 & & 8 \\
\hline neuroepithelial & & & $73(5.3)$ \\
\hline optic nerve glioma & 34 & & 34 \\
\hline meningioma & 42 & 97 & $139(10.1)$ \\
\hline mesenchymal & & & $45(3.3)$ \\
\hline rhabdomyosarcoma & 17 & 21 & 38 \\
\hline lipoma & 3 & & 3 \\
\hline liposarcoma & 3 & & 3 \\
\hline inflammatory & & & $98(7.1)$ \\
\hline nonvasculitic & 58 & & 58 \\
\hline vasculitic, nongranulomatous & 23 & & 23 \\
\hline carcinoma & & & $311(22.6)$ \\
\hline cystic & & & $166(12.1)$ \\
\hline other & & & $317(23)$ \\
\hline
\end{tabular}

* Adapted from Henderson.

matory reaction caused by the surgical procedure helps to induce resorption of the lesion. Clearly, it is important to differentiate benign lesions of this type from the cystic changes of more sinister lesions, such as hemangiomas of the bone, giant cell tumor of bone, or osteogenic sarcoma. These lesions are definitively treated surgically.

Osteogenic sarcoma is rarely found in the orbital region. It may occur more frequently in cases in which there are genetic defects and a history of radiation therapy usually in the setting of an irradiation-treated retinoblastoma. ${ }^{3}$ Even radical excision may not improve the poor prognosis in patients with these tumors.

The periosteal lining of the orbit, also known as the periorbita, is an important structure in the orbit because it helps serve as a barrier to the intracranial extension of neoplastic growth. The periorbita proper can also be involved in nonneoplastic lesions; in young children, this can be the site of an idiopathic inflammatory condition known as infantile cortical hyperostosis (Caffey disease). ${ }^{6}$

The only cartilaginous structure of the orbit is the trochlea, a U-shaped structure that suspends the tendon of the superior oblique muscle. Tumors arising from the trochlea such as chondromas and chondrosarcomas are exceedingly rare.

\section{Lesions of Vascular Origin}

Vascular lesions of the orbit include capillary hemangi-

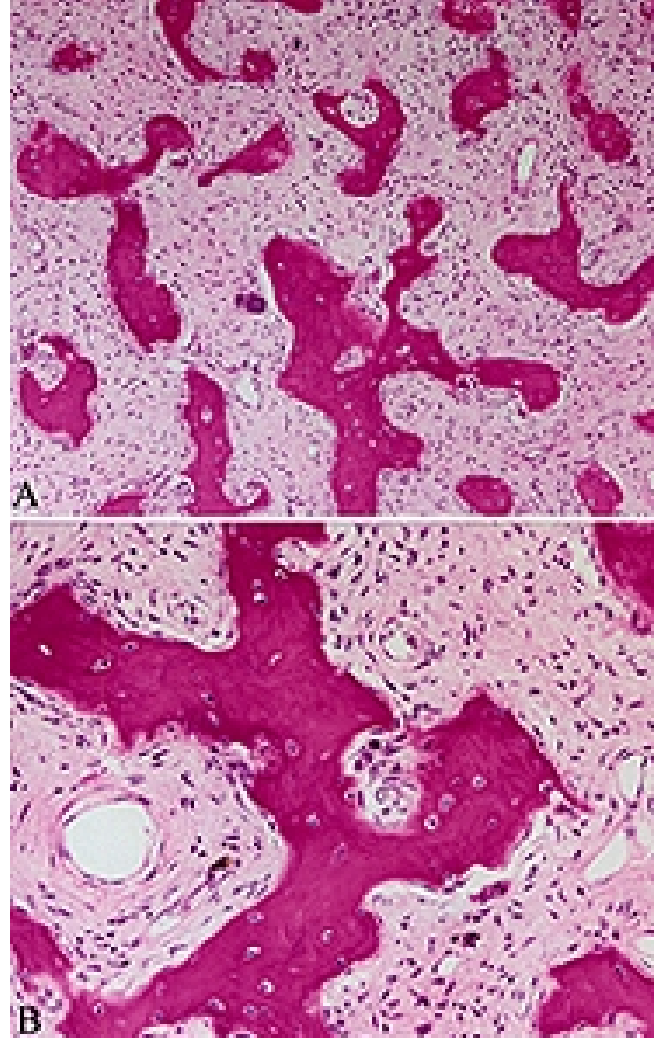

Fig. 1. A fibrous dysplastic lesion was obtained in the orbit of a 40-year-old woman. Photomicrograph showing spindle-cell proliferation and metaplastic bone formation (A). A high-magnification photomicrograph emphasizing the absence of osteoblastic reaction of the bony trabeculae (B). $\mathrm{H} \& \mathrm{E}$, original magnification $\times 100$ (A) and $\times 200(B)$.

omas, cavernous hemangiomas, lymphangiomas, and hemangiopericytomas, as well as AVMs of intraorbital vessels. Together, these lesions comprise 12 to $15 \%$ of orbital tumors. ${ }^{3}$ Capillary hemangiomas usually present by the age of 6 months. These lesions are benign, yet widely infiltrative, and they can be associated with cutaneous manifestations such as strawberry nevi. Spontaneous resolution usually occurs over a 3- to 5-year period, but in some cases there may be significant residual cosmetic disfigurement and amblyopia. They can be treated with argon laser therapy or corticosteroid therapy to promote involution.

Cavernous hemangiomas, the most common benign orbital tumors, are found primarily in young and middleaged adults. These are low-flow circumscribed lesions usually situated behind the globe, most commonly within the muscle cone. They do not usually interfere with visual acuity, except when the tumor lies in the orbital apex, where it may affect the optic nerve. Cavernous hemagiomas are readily amenable to surgical treatment and do not recur. They are seen as well-delineated enhancing masses on CT scans (Fig. 2). On $\mathrm{T}_{1}$-weighted MR images, these lesions have a low-intensity signal (Fig. 3 upper), whereas they are hyperintense on $\mathrm{T}_{2}$-weighted MR images (Fig. 3 lower). In the event of intralesional thrombosis, a higher-intensity signal will be demonstrated on $\mathrm{T}_{1}$-weighted MR images. ${ }^{\text {? }}$ 


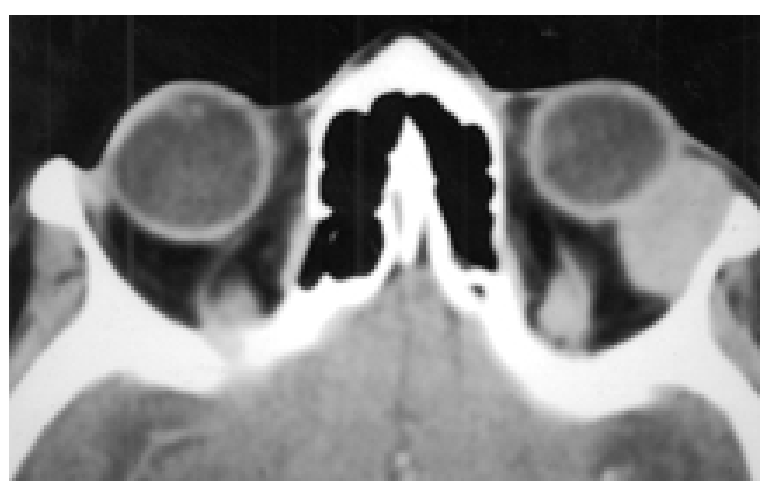

Fig. 2. Postcontrast axial CT scan obtained in a patient with a cavernous hemangioma of the left orbit.

Lymphangiomas are slow-growing lesions that may occur in the orbit even though the orbit does not have a lymphatic drainage system. These lesions most commonly occur in children and young adults and may cause a slowly progressive exophthalmos. Hemorrhage into the tumor may cause rapid expansion of the orbital contents and subsequent rapidly progressive, worrisome exophthalmos. These episodes may resolve spontaneously. Lymphangiomas are exceedingly difficult to manage surgically because they involve critical orbital structures, and laser therapies are currently being used as adjuvants when

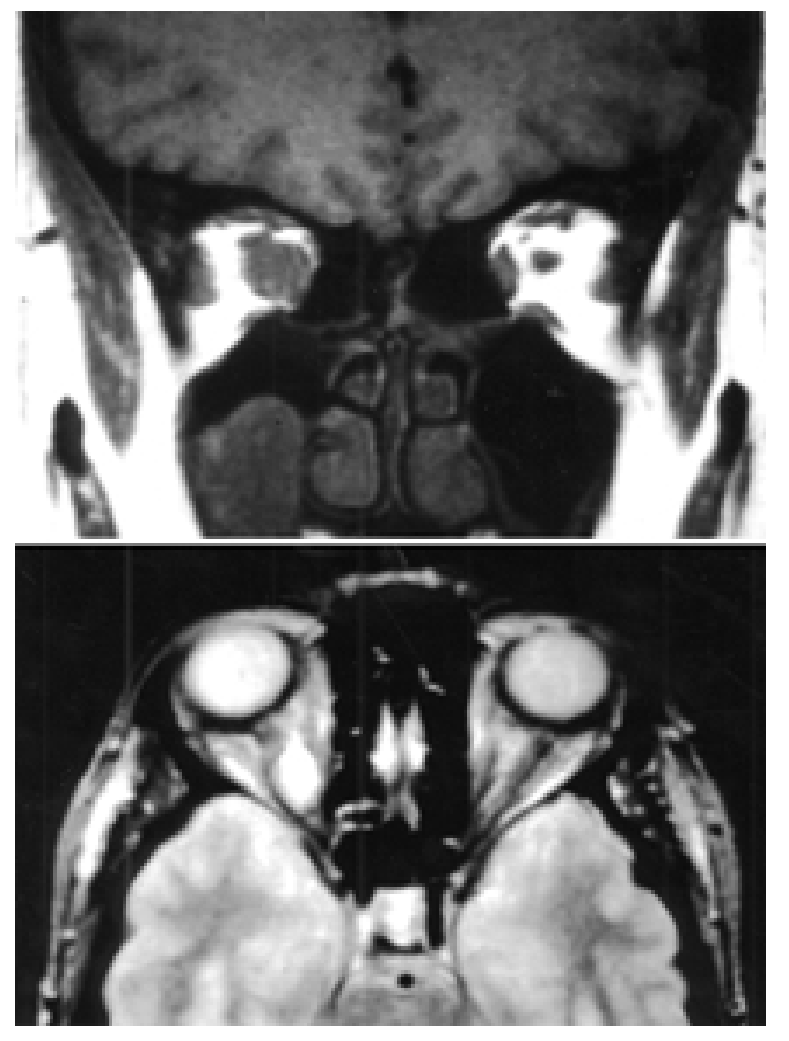

Fig. 3. Upper: Coronal $\mathrm{T}_{1}$-weighted $\mathrm{MR}$ image revealing a hypointense mass in the right orbit consistent with an orbital hemangioma. Lower: Axial $\mathrm{T}_{2}$-weighted axial $\mathrm{MR}$ image obtained in the same patient. treatment is necessary. Because of their repeated episodes of hemorrhage, they demonstrate a heterogeneous signal intensity on $\mathrm{T}_{1}$ - and $\mathrm{T}_{2}$-weighted MR imaging ${ }^{7}$ as a result of the presence of blood breakdown products. On MR imaging these lesions are not as enhancing as hemangiomas and may not enhance at all.

Hemangiopericytomas are malignant tumors thought to arise from undifferentiated mesenchymal cells with pericytic differentiation (Fig. 4). They are most common in young and middle-aged adults. They are invasive tumors with a known propensity for metastasis, although this is rare from the orbit. Total excision is necessary to prevent recurrence.

\section{Miscellaneous Tumors Of Mesenchymal Origin}

Other tumors of mesenchymal origin worthy of mention include rhabdomyosarcoma and fibrous histiocytoma. The former are the most common malignant orbital tumors affecting children. They are rapidly progressive lesions that respond readily to irradiation and chemotherapy and therefore should not be missed. On CT scans, they appear as bulky, mildly enhancing masses that erode bone, and they can invade into the surrounding sinuses. On MR images, they are of intermediate signal intensity.

Fibrous histiocytomas are the most common orbital tumors in adults. They develop insidiously and can be local-

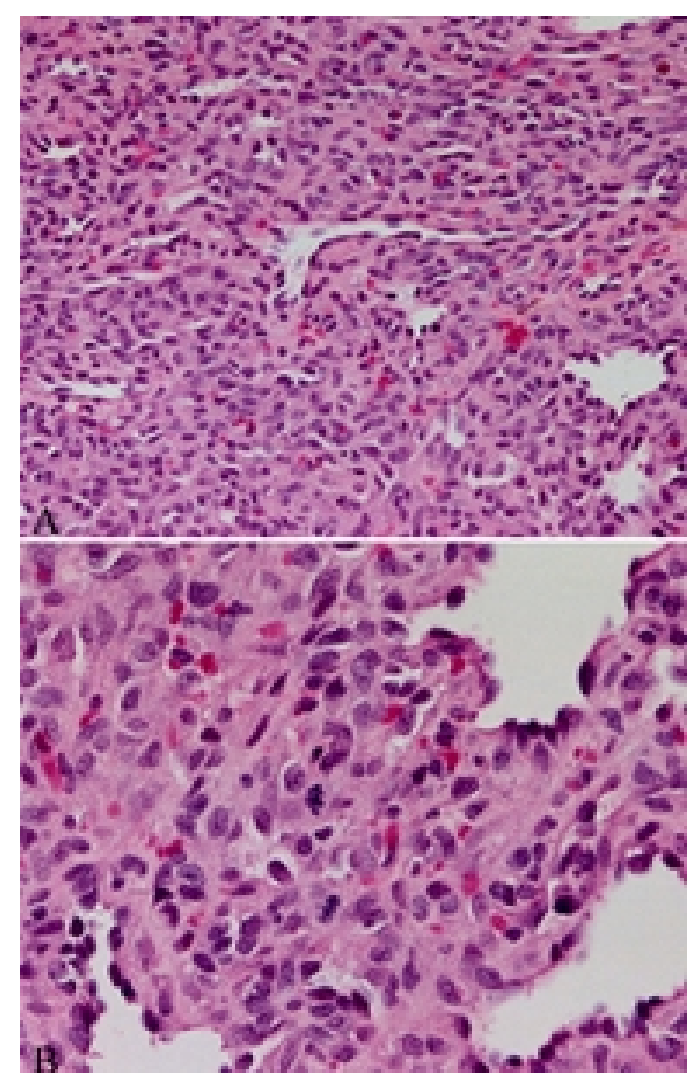

Fig. 4. Photomicrographs. An orbital hemangiopericytoma obtained in a 56-year-old woman. A: The tumor is characterized by numerous vascular channels intermixed with plump, polygonal cells with mild nuclear atypia $B$ : Mitotic figures may be prominent. $\mathrm{H} \& \mathrm{E}$, original magnification $\times 100(A)$ and $\times 200(B)$. 
ly infiltrating, and although they are benign lesions, their rate of recurrence is high. They should be resected, and the resection margins should be wide. Malignant fibrous histiocytomas may metastasize and lead to death.

Although the rectus, superior and inferior oblique, and levator palpebrae muscles may give rise to fibromas, fibrosarcomas, leiomyomas, and leiomyosarcomas, these primary muscle tumors are exceedingly rare. Lipomas, which can originate from the voluminous orbital fat, are sometimes difficult to differentiate clinically and pathologically from normal fat.

\section{Tumors Arising From Neural Structures and Their Coverings Within the Orbit}

Nerve sheath tumors within the orbit can give rise to neurofibromas, schwannomas, and malignant peripheral nerve sheath tumors, comprising approximately $15 \%$ of orbital tumors. ${ }^{3}$ These lesions do not involve the optic nerves because the optic nerve lacks Schwann cells; rather, they develop from the peripheral motor nerves that supply extraocular muscles, the first and second division of the trigeminal nerve, or from sympathetic or parasympathetic fibers. Neurofibromas can be subdivided into three classes: solitary, diffuse, and plexiform. Solitary neurofibromas are single, encapsulated lesions that are not associated with NF. Because these lesions are well circumscribed, complete resection is possible. In contradistinction, diffuse neurofibromas, which can be associated with NF, are not solitary lesions, and they may involve orbital tissues in a more extensive manner. Plexiform neurofibromas are pathognomonic of NF1. These extensive lesions involve bundles of nerves, each with perineuriumencased tumor, making total resection impossible. Subtotal resection is performed when the mass of tumor tissue produces cosmetic deformity. Patients with NF may harbor plexiform neurofibromas as well as optic nerve gliomas and meningiomas.

Schwannomas, also known as neurilemmomas, are tumors consisting exclusively of Schwann cells. These lesions grow slowly, allowing the eye to accommodate a severe degree of exophthalmos (Fig. 5). The lesion is solid, and it is encapsulated by the perineurium of the nerve from which it arose; because of these features, it is amenable to total resection. Schwannomas are unlikely to undergo malignant degeneration. Malignant peripheral nerve

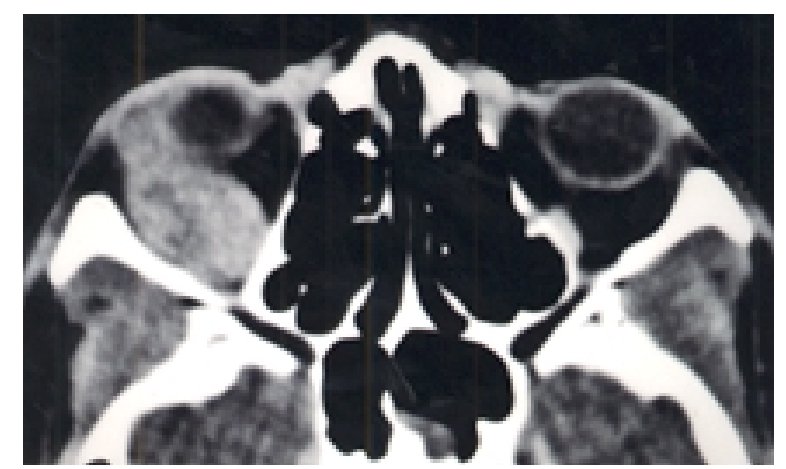

Fig. 5. Postcontrast axial CT scan obtained in a patient with a schwannoma involving the right orbit. sheath tumors (previously termed neurofibrosarcomas or malignant schwannomas) are very rare lesions, but they are known to be associated with NF1. These malignant lesions must be distinguished histopathologically from mesenchymal tissue tumors.

Paragangliomas (chemodectomas) can arise from the ciliary ganglion, although they are more typically seen around the carotid arteries and in the jugular fossa (growing from the ganglia of the vagus nerve). In general, nerve sheath tumors exhibit a low-intensity signal on $\mathrm{T}_{1}$-weighted and high-intensity signal on $\mathrm{T}_{2}$-weighted $\mathrm{MR}$ images. ${ }^{7}$ Imaging of plexiform neurofibromas may demonstrate involvement and enlargement of the eyelids, fat, extraocular muscles, and lacrimal gland, as well as some facial deformity. Solitary neurofibromas and schwannomas are indistinguishable radiologically, as both appear as solitary, ovoid masses resembling cavernous hemangiomas.

The optic nerve is a white matter tract of the brain. Masses formed by abnormal proliferation of fibrillary astroglial cells in this area are termed optic nerve gliomas, which are the second most common orbital tumor in children, the fifth most common in adults, and the most common in patients with NF1. Most optic nerve gliomas are low-grade lesions and are most likely pilocytic astrocytomas; in approximately $50 \%$ of cases these lesions are diagnosed in patients before the age of 5 years. ${ }^{6}$ Patients with optic nerve gliomas typically present with exophthalmos, and examination shows a severely decreased visual acuity, with some patients being completely blind in the affected eye. Papilledema and pallor of the optic disc are also common findings. Optic gliomas are best visualized on MR imaging, which can demonstrate extraorbital extension, as well as a fusiform enlargement of the optic nerve. When these lesions are restricted to the orbit, they usually prove isointense with white matter on both $\mathrm{T}_{1}-$ and $\mathrm{T}_{2}$-weighted MR images whereas lesions that involve the chiasm may have increased signal intensity on $\mathrm{T}_{2}$-weighted MR images. ${ }^{7}$

Meningiomas are thought to arise from arachnoid cap cells, and therefore, they generally follow the distribution of the meninges (Fig. 6). Orbital meningiomas can be subdivided into primary and secondary types; the former arise in the orbit and the latter expand to include the orbit. They comprise approximately $10 \%$ of all intraorbital tumors, with a female/male preponderance of $3: 1 .^{3}$ Patients with orbital meningiomas present with a wide range of signs and symptoms, most commonly exophthalmos, decreased visual acuity, pallor of the optic disc, or visual field deficits. Unilateral boggy edema of the eyelid, resembling myxedema, is a rare, peculiar finding in cases of longstanding meningiomas. Orbital pain or headache is an uncommon symptom in patients with these lesions. Computerized tomography scanning may demonstrate calcification in patients with meningiomas (Fig. 7). On $\mathrm{T}_{1}-$ weighted MR images, meningiomas are usually isointense with muscle, whereas on $T_{2}$-weighted MR images, they are variable. These lesions enhance markedly after administration of gadolinium. In general, angiography can determine the site of origin of these tumors by demonstrating their blood supply. Meningiomas originating from the optic nerve sheath are particularly slow growing. In the preimaging days they were often mistaken for "chronic optic neuritis." 


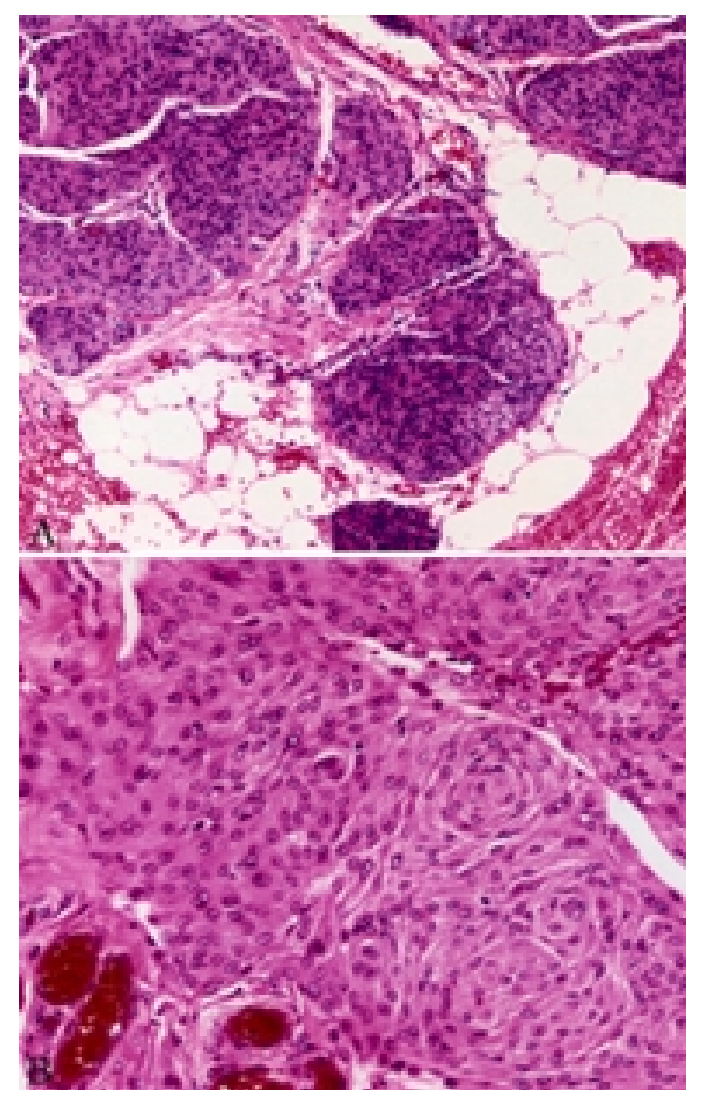

Fig. 6. Photomicrographs. A transitional meningioma infiltrating the adipose periorbital tissues obtained in a 38-year-old woman (A). The typical meningothelial cells with bland nuclei and syncytial cellular arrangement (B). The meningioma diffusely infiltrated the orbital bony structures and the temporalis muscle. $\mathrm{H} \& \mathrm{E}$, original magnification $\times 100(\mathrm{~A})$ and $\times 200(\mathrm{~B})$.

\section{Dermoids and Epidermoids}

Dermoids and epidermoids are benign cystic lesions that arise from congenital rests. Most orbital dermoids are located anteriorly, becoming evident during childhood because of their superficial location (Fig. 8 upper). The most frequent location is the frontozygomatic suture (Fig. 8 lower). Growth usually results from the slow production

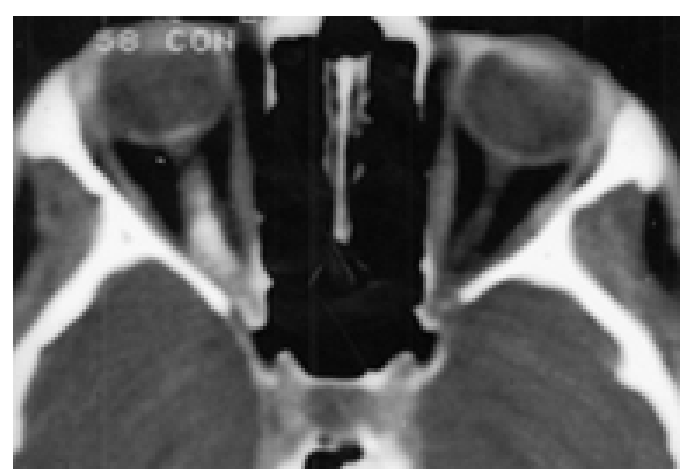

Fig. 7. Noncontrast axial CT scan obtained in a patient with right optic nerve sheath meningioma, revealing the presence of tumoral calcifications. and accumulation of dermal products within the mass. On CT scans, these lesions appear well delimited from the surrounding structures, have smooth margins, and can have a fluid level (Fig. 9).

\section{Metastatic Lesions}

There is a variety of metastatic tumors (Fig. 10). The patient's age at presentation helps differentiate among the possible tumor types. In children, the most common metastatic lesions include neuroblastoma, Ewing tumor, chloroma, and Langerhans cell histiocytosis. Neuroblastomas in pediatric patients are the most common metastatic tumor to spread to the orbit and have been reported in up to $40 \%$ of patients. ${ }^{4}$ These tumors arise from the adrenal medulla or parasympathetic or sympathetic structures, often spreading to both orbits, where they frequently cause an abrupt onset of exophthalmos and bilateral eyelid edema and ecchymosis (Fig. 11). Ewing tumor is a malignant bone lesion that classically involves the limbs, ribs, or pelvis. When this tumor metastasizes to the orbit, it usually presents as an abrupt hemorrhagic exophthalmos. Chloromas are the extramedullary form of acute myelogenetic leukemia. This lesion is also heralded by the onsets of acute hemorrhagic exophthalmos. Langerhans cell histiocytosis is a term used to describe three similar but separate multisystem diseases: eosinophilic granulomatosis (or histiocytosis X), Hand-Schüller-Christian disease, and Letterer- Sewe disease. Only the first two subclasses

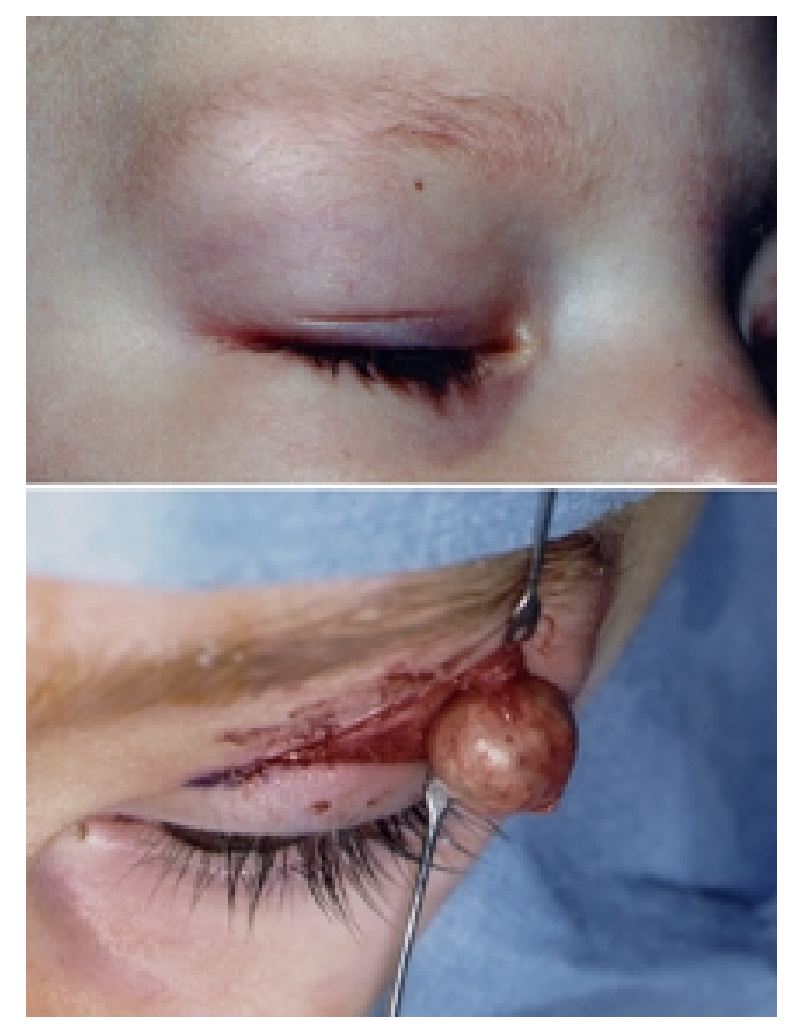

Fig. 8. Upper: Photograph showing upper-lid swelling in a patient with a frontal dermoid involving the orbit. Lower: Macroscopic view of a dermoid of the orbit during surgical removal. Note the typical location in the superotemporal orbit. 


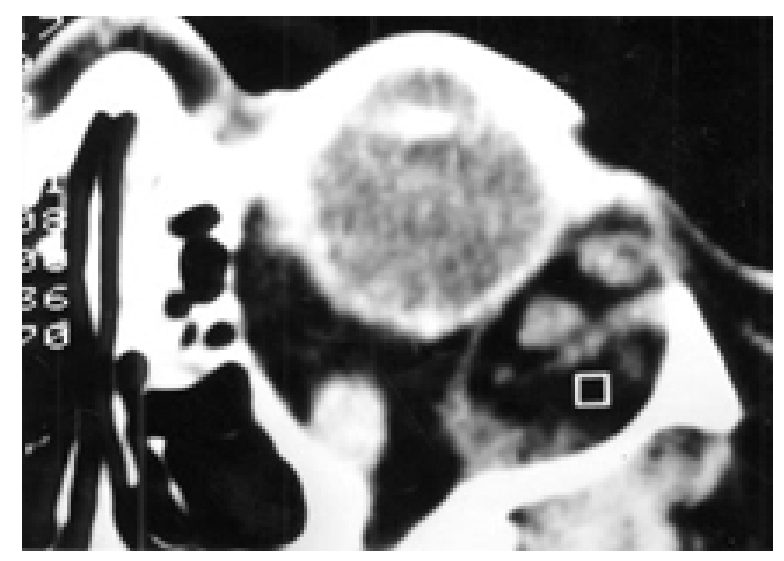

Fig. 9. Axial nonenhanced CT scan of the orbit obtained in a patient with a dermoid of the left orbit.

cause orbital disease: eosinophilic granulomatosis with solitary granulomatous proliferation may occur in the orbit, causing painful eye swelling and exophthalmos, whereas Hand-Schüller-Christian disease has exophthalmos as part of its clinical triad, as well as diabetes insipidus and lesions of the bone.

In adults, the most common primary tumors to metastasize to bone are, in decreasing order, breast, lung, prostate, melanoma, gastrointestinal tract, and kidney. Because the orbital tumor may be the presenting lesion in up to $25 \%$ of cases, ${ }^{1}$ it should be strongly considered in the differential diagnosis of adult patients with orbital lesions causing exophthalmos. Due to the associated abysmal overall median survival rates of 1 to 2 years postdiagnosis, ${ }^{1}$ these tumors are best treated with radiation therapy or chemotherapy. Obviously, treatment decisions must be modified according to the degree of systemic involvement and prognosis in each patient.

\section{CLINICAL PRESENTATION}

\section{Exophthalmos as a Presenting Sign}

Careful assessment of clinical signs and symptoms and their chronology and various associations helps to differentiate the various disease processes that present as spaceoccupying masses. The cardinal sign suggestive of orbital tumor is eye exophthalmos, or proptosis. Displacement of the globe horizontally or vertically is often referred to as dystopia and may suggest a particular location within the orbit.

In the evaluation of globe protrusion, distinguishing between pseudexophthalmos and exophthalmos is essential. In the ipsilateral eye, lid retraction such as in Graves disease may give the appearance of exophthalmos, as can congenital glaucoma and unilateral high myopia. Features of the contralateral eye such as microphthalmos due to a congenital abnormality may give the examiner a false impression of exophthalmos; the same is true for enophthalmos caused by various processes, including blow-out orbital fractures, inflammatory processes, and orbital involvement of metastatic scirrhous adenocarcinomas, usually originating in the breast. Congenital disease-related craniofacial asymmetries may also give the false impres-

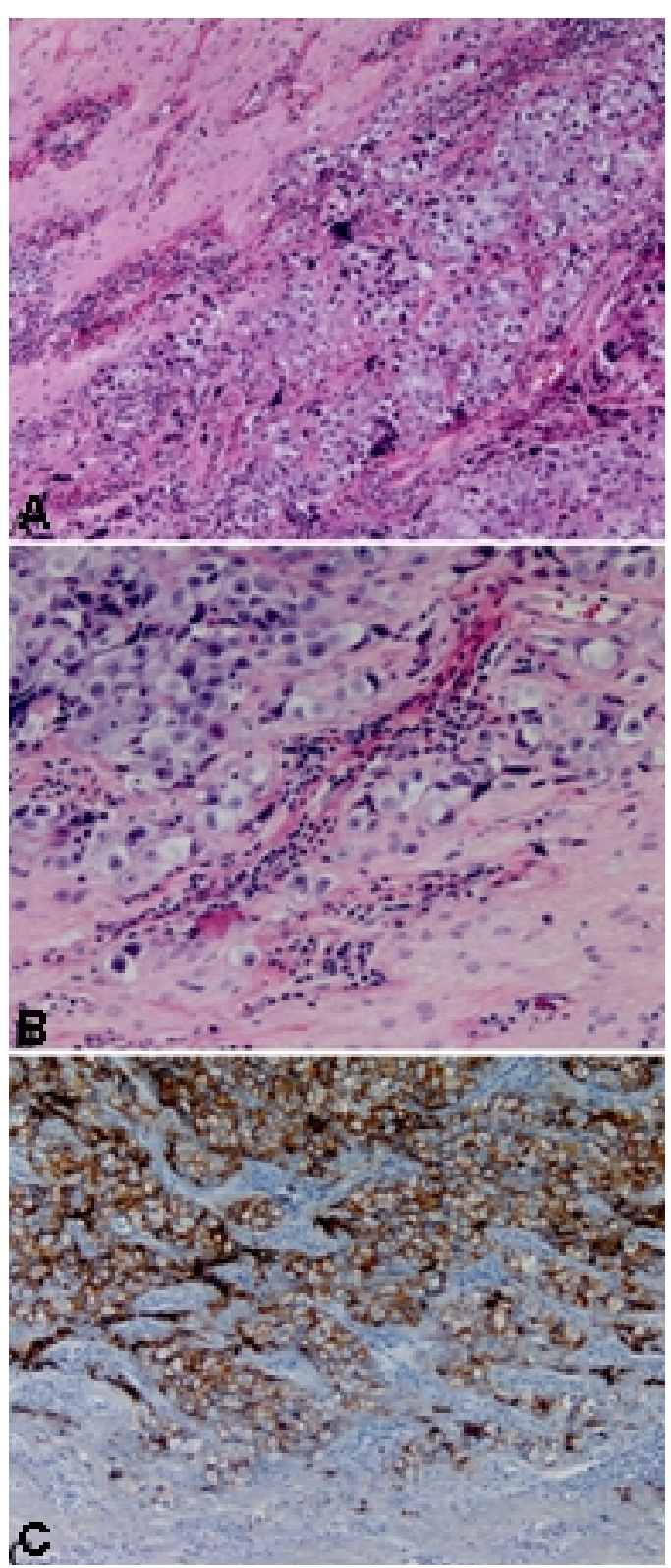

Fig. 10. Photomicrographs. A male patient with a germinoma of the suprasellar region presented with visual difficulties. A: The optic chiasm and optic nerve were diffusely infiltrated by the germinoma. Large polygonal cells with large nuclei and prominent nuclei permeated the entire optic nerve. B: Lymphocytic inflammatory infiltrate accompanied the neoplastic cells. C: An immunohistochemical stain for placental alkaline phosphatase confirmed the germinal nature of the tumor. A and B: H \& E, original magnifications $\times 100(\mathrm{~A}), \times 200(\mathrm{~B})$, and $\times 100(\mathrm{C})$.

sion of exophthalmos. True exophthalmos may be associated with those craniofacial abnormalities (for example, those found in Crouzon disease and Apert syndrome) associated with midface hypoplasia. An accurate diagnosis will be assisted by a strong degree of suspicion of pseudexophthalmos, careful assessment of patient history, and thorough physical examination in which emphasis is placed on visual inspection; if available, an exophthalmometer will also aid in diagnosis. 


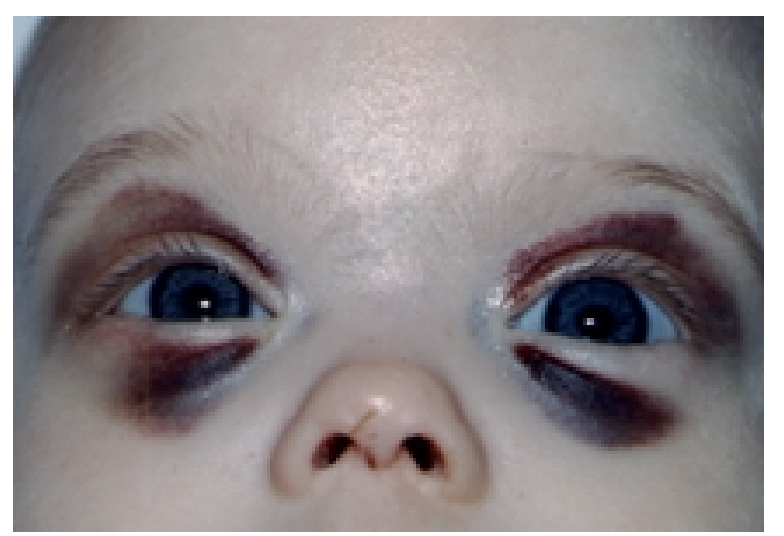

Fig. 11. Photograph of bilateral ecchymosis in a child with metastatic neuroblastoma.

Several features of the history, such as unilateral compared with bilateral eye involvement, direction of displacement, patient age, and the rate of symptom onset may help establish the nature of the disease. Bilateral globe protrusion is more likely in cases of systemic disease such as dysthyroid orbitopathy, and this is usually easily distinguished from unilateral orbital tumors. In the less common cases in which a thyroid origin results in unilateral exophthalmos, retraction of the eyelid can help identify this origin, as can a detailed clinical history, physical examination, and laboratory thyroid function tests. A discussion of dysthyroid orbitopathy will not be covered in this article, as it is not a true tumor and its relevance to the surgeon is limited to differential diagnosis and to the rare cases in which myxedema is severe enough to warrant surgery for corneal exposure or compressive optic neuropathy. Bilateral exophthalmos may occasionally be a feature of certain orbital tumors such as lymphoma, histiocytoma, or metastatic lesions.

Etiopathological information may be inferred from the tumor mass-induced direction of global deviation. With rare exceptions, tumor growth displaces the globe away from the lesion. Tumors of the lacrimal gland, for example, classically induce medial and inferior displacement of the globe. Tumors of the optic nerve generally cause displacement of the globe along the axis of the orbit, whereas tumors arising from the extraocular muscles tend toward axial and slightly inferior displacement of the eye.

The patient's age of onset of the exophthalmos is also important because the incidence of certain lesions is different in the pediatric and adult populations. Infants and neonates with congenital, often subtle exophthalmos, are more likely to harbor teratomas, dermoid cysts, and capillary hemangiomas than the slightly older early childhood group in which the children are most likely to harbor lyphangiomas, optic gliomas such as pilocytic astrocytomas, or rhabdomyosarcomas. Lacrimal gland tumors, metastases to the orbit, cavernous hemangiomas, and neurilemmomas are more likely to occur in adults.

In general, a lesion that grows insidiously is more likely benign, whereas a more rapid process indicates a more aggressive histological type. Hyperacute onset of exophthalmos is more likely to be caused by inflammatory processes than tumor growth, and this should help guide investigations.

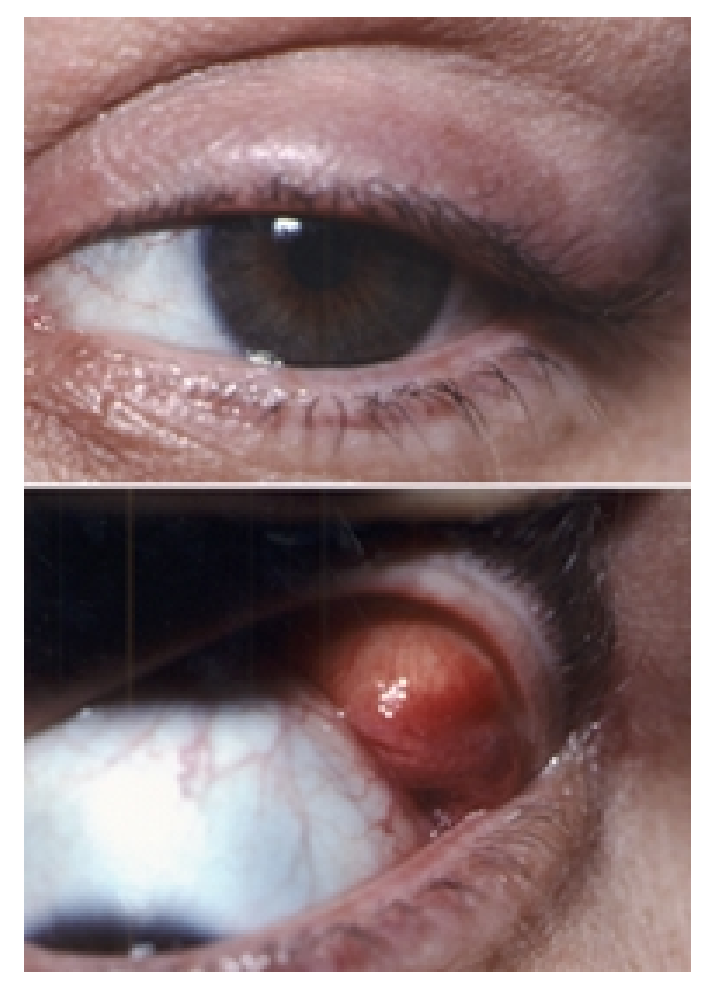

Fig. 12. Photograph showing upper-lid swelling (upper) and orbital lymphoma visible on inspection.

\section{Signs and Symptoms Other Than Exophthalmos}

The rate and extent of visual acuity deterioration is of great importance in distinguishing primary from secondary involvement of the optic nerve. An optic nerve glioma or nerve sheath meningioma tends to cause profound visual deterioration, whereas a tumor that is external to the nerve affects visual acuity by compression and causes a mild visual deficit unless it is very advanced and is exerting considerable mass effect.

Pain is commonly a feature of inflammatory diseases or infections such as myositis or dacryoadenitis, although metastases and rapidly expanding neoplastic processes may also cause symptomatic pain. Orbital lesion-induced pain tends to be localized, constant, and may be more prominent at night. The sudden onset of orbital pain in a child may be caused by a hemorrhage into a cystic lymphangioma. Processes causing pain that can be localized deep in the orbit suggest orbital apex involvement, especially when there is ophthalmoplegia and/or facial dysesthesias in the distribution of $\mathrm{V}_{2}$. Benign tumors of the orbit usually tend not to cause any pain, although a feeling of pressure in the orbit is typically reported in cases of slowgrowing lesions.

There are two mechanisms by which a tumor can cause diplopia: first, by infiltrating the nerves supplying the extraocular muscles, which is seen with malignant tumors and metastases; and second, by restricting the normal extraocular motility function and/or deviating the axis of the eye. A sudden onset of normal extraocular motility loss is usually associated with an aggressive malignant lesion.

Changes in the size of the pupils can be observed in 
cases in which the tumors invade or compress the parasympathetic (third nerve through the ciliary ganglion) or the sympathetic fibers innervating the pupillary dilators. This is found in cases of chemodectomas (paragangliomas), but many tumor types can also cause anisocoria through involvement of the sympathetic or parasympathetic fibers supplying the iris dilator and pupillary sphincter.

Bruits may occur with AVMs and may be a source of distress to patients, who may describe constant or intermittent sounds that are pulsatile or swishing in nature. They may also have a positional component, being exacerbated with recumbency. This symptom is associated with arteriovenous fistulas, and more often of traumatic origin.

A variety of fundus changes can be observed in association with orbital tumors. Optic atrophy, which may be preceded by edema, is characteristic of long-standing optic nerve damage. Compressive lesions that impede drainage though the central retinal vein can cause optociliary shunts, which consist of enlarged preexisting peripapillary vessels that divert blood from the central retinal venous circulation to the peripapillary choroidal circulation when obstruction of the normal drainage channels occurs. These can be demonstrated in patients with optic nerve sheath meningiomas, although they are seen most commonly with central retinal vein occlusion. Choroidal folds are a series of parallel, alternating light and dark striae, which are a common manifestation of a variety of lesions including orbital tumors. They tend to be more common when the globe is directly compressed, especially by anteriorly placed tumors, but at times they can precede the occurrence of clinically evident exophthalmos. Venous dilation can be associated with disc edema in patients with orbital masses when the optic nerve is compressed.

Anteriorly located tumors can present with eyelid swelling (Fig. 12 upper) and can be visible on ispection (Fig. 12 lower).

\section{NEUROIMAGING FEATURES}

In the $\mathrm{CT}$ and MR imaging era, plain radiography has a limited role in the diagnostic workup of patients with suspected orbital tumor. Before the availability of high-resolution cross-sectional imaging, ultrasonography was very popular. Ultrasonography is, however, still useful in the evaluation of ocular masses and fluid-filled orbital tumors such as cysts and vascular lesions.

Computerized tomography scanning is the imaging modality of choice for bone lesions, because of the natural contrast afforded by the retrobulbar fat, bone, and air. Orbital imaging protocols must include coronal as well as axial slices. In general, 1- to 5-mm-thick slices are adequate for evaluation of orbital masses. Intravenous contrast administration helps in the characterization of individual lesions.

Magnetic resonance imaging superbly demonstrates the anatomy of the orbit is sensitive to the different soft-tissue characteristics of orbital lesions. Additionally, MR imaging has the advantage of being a nonradiation-based modality, and, as such, does not deliver radiation to the lens. Despite the availability of MR angiography, conventional angiography of orbital lesions remains the gold standard for detecting vascular lesions, such as AVMs and low- flow dural arteriovenous fistulas, and for defining the extent of the blood supply to orbital tumors, especially meningiomas. Cerebral angiography should be considered for any patient with pulsatile exophthalmos.

The variations in imaging characteristics of all different orbital lesions are diverse and exceed the scope of this review. Furthermore, it should be emphasized that diagnoses of neoplastic growth should not be established in the absence of histological evaluation of a biopsy specimen. In this review, we focus on characteristics that can be useful to distinguish the different classes of lesions and illustrate imaging features of common lesions. As a general rule, benign tumors cause displacement and molding of adjacent structures, whereas malignant lesions display an aggressive pattern of growth, destroying and obliterating the contours of surrounding structures. On imaging studies, these two patterns of growth are usually, albeit not always, distinguishable. In cases of primary orbital tumors the extensive bone destruction produced by metastatic lesions is rarely demonstrated.

The radiological findings in pseudotumor depend on the structures and the nature of the ongoing inflammatory process, but the following generalities can be made. Pseudotumors are enhancing lesions that are usually isodense with muscle on $\mathrm{T}$-weighted images, whereas they are nearly isodense with fat on $\mathrm{T}_{2}$-weighted images. ${ }^{7}$ Myositic pseudotumor may be seen as an enlargement of one or more muscle bellies in the orbit, but it may also involve the tendinous origin, unlike dysthyroid orbitopathy.

Dysthyroid orbitopathy, which results in extraocular muscle infiltration with lymphocytes, plasma cells, mast cells, and mucopolysaccharides may involve any muscle of the orbit but most commonly enlarges the inferior or medial rectus. This can be helpful in distinguishing Graves disease from neoplastic growth, whereas the sparing of the tendinous origin in Graves disease distinguishes it from the myositic variant of pseudotumor.

Noteworthy imaging characteristics include enlargement of the optic canal observed in lesions involving the optic nerve; destruction of bone can occur in cases of aggressive tumors such as rhabdomyosarcomas. A decrease in size of the canal can be observed in the presence of nonneoplastic lesions involving the bone such as fibrous dysplasia and ossifying fibroma.

Arteriovenous malformations are associated with the presence of phleboliths, (calcified deposits in a vein) which appear almost isodense with bone on CT scans. ${ }^{7}$ This type of lesion is also suggested by the presence of flow voids demonstrated on MR imaging. Calcium deposits in the mass of a lesion are also more suggestive of certain tumor types, most notably retinoblastoma and meningioma.

\section{PRINCIPLES OF TREATMENT}

The best strategy for managing orbital tumors is largely determined by the nature of the lesion. Lesions best suited to medical treatment include the dysthyroid orbitopathies, infectious processes involving the orbit, such as mild cases of cellulitis, and inflammatory pseudotumors. Definitive surgical treatment remains the mainstay of therapy for the majority of symptomatic orbital tumors. The type of anesthesia and the most appropriate surgical 


\section{Overview of orbital tumors}

approach to the lesion are dependent on its anatomical location, proximity to vital structures, and extent of involvement as visualized on CT scanning and MR imaging. Orbital and nasal approaches can be warranted, especially when the tumor does not involve the apex or extend beyond the confines of the orbit.

Orbital approaches can be made more extensive when the lesion is posterior to the equator of the eye. A lateral orbitotomy, with temporary removal of the lateral orbit wall, may provide excellent exposure of the orbital contents, allowing for tumor resection without damage to the extraocular muscles or their nerve supply. This technique is useful for lesions in the lacrimal fossa and lateral orbit, including the areas above and below the optic nerve.

The transcranial approach is useful for the resection of medially situated lesions, especially those situated at the orbital apex or those involving the optic nerve. In this approach, it is important to establish the surgery-related goals preoperatively. Certainly, with a malignant process confined to the orbit, cosmesis is secondary to controlling the spread of disease, and it is important to attempt to determine the nature of the lesion prior to undertaking the operation.

Orbital exenteration is the excision of the eyelids and the removal of the conjunctiva, globe, optic nerve, extraocular muscles, the lacrimal gland, and all the soft tissues of the orbit. Careful consideration of a patient's ultimate prognosis should precede this surgical procedure.

\section{CONCLUSIONS}

Orbital tumors encompass a heterogeneous variety of lesions. Careful evaluation of a patient's history and examination along with modern high-resolution imaging studies provide invaluable information regarding the possible origin of an orbital lesion. For most tumors, surgery is the treatment of choice.

\section{References}

1. Dutton JJ, Byrne SF, Proia AD: Diagnostic Atlas of Orbital Diseases. Philadelphia: WB Saunders, 2000

2. Henderson JW: Orbital Tumors, ed 3. New York: Raven Press, 1994

3. Housepian EM, Trokel SL, Jakobiec FA, et al., in Youmans JR (ed): Neurological Surgery, ed 3. Philadelphia: WB Saunders, 1990, Vol 5, pp 3371-3411

4. Kanski JJ: Clinical Ophthalmology: A Systematic Approach, ed 4. Oxford: Butterworth-Heinemann, 1999

5. Laws ER Jr (ed): The Diagnosis and Management of Orbital Tumors. New York: Futura Publishing, 1988

6. Shields JA: Diagnosis and Management of Orbital Tumors. Philadelphia: WB Saunders, 1989

7. Zimmerman RA, Gibby WA, Carmody RF: Neuroimaging: Clinical and Physical Principles. New York: Springer-Verlag, 2000

Manuscript received April 23, 2001.

Accepted in final form May 3, 2001.

Address reprint requests to: Giuseppe Lanzino, M.D., Department of Neurosurgery, Box 800212 University of Virginia Health Sciences Center, Charlottesville, Virginia 22908. email:glanzino@ hotmail.com. 